\title{
Simulation as an educational strategy to acquire surgical skills
}

\author{
La simulación como estrategia educativa para adquirir habilidades quirúrgicas
}

\author{
Jesús Tapia-Jurado ${ }^{*}$ and Pamela Soltero-Rosas² \\ ${ }^{1}$ General Surgeon, Chairman of the Medical Academy of Surgery; ${ }^{2}$ Medical intern on social service, Faculty of Medicine, UNAM. Mexico City. Mexico
}

Surgery is a branch of medicine that over the last 50 years has had a highly accelerated evolution and has become less traumatic and mutilating. However, due to the appearance of new endoscopic procedures, together with limitations in terms of equipment and innovative instruments, the learning of minimally invasive surgical abilities and skills has not achieved the safety it requires. The use of medical services entails a high risk that involves a mortality of 1 in 1000 cases per year; in contrast, commercial airline services add up to 1 in one million. On the other hand, medical error was reported to be the third cause of death in 2016, only below problems of cardiac origin and cancer ${ }^{1}$. For this reason, today it is no longer possible to learn exclusively in front of the patient, but there is a search for educational strategies that favor an adequate learning of surgery with minimal risk to patients.

According to a study published by Mattar $^{2}, 21 \%$ of residents are not ready for operating, 30\% are not able to perform a laparoscopic cholecystectomy by themselves, $24 \%$ do not early identify signs of complications, $30 \%$ are not able to manipulate tissues in an atraumatic way, $26 \%$ do not recognize anatomical planes and $56 \%$ do not adequately carry out suture procedures. On the other hand, schedule restrictions start to exist for residents, and they have increasingly less exposure to patients and to surgical procedures. All this has led to the search for educational strategies that enable the acquisition of surgical abilities and skills in models other than patients. For this reason, simulation has emerged as an educational strategy in medicine, which is a training methodology that reproduces reality in a controlled environment and the purpose of which is to acquire and improve professional, communication, coordination, leadership and, especially, psychomotor competences ${ }^{3}$.

Simulation for the acquisition of surgical skills has the following objectives: practicing all the skills demanded by the professional curriculum, standardize the indispensable surgical procedures from an educational point of view, repeat the practices as many times as necessary and, consequently, increase patient safety. The benefits of simulation are: learning in a controlled environment, free of risks and adverse consequences for the patient; errors that can occur and that are unacceptable in clinical practice with the patient, can be allowed in a simulation, analyzing and discussing the error and offering the opportunity for its correction ${ }^{4}$; it decreases the time of student's learning curve; and economic resources, materials and operating room equipment are optimized and saved.

It is important to remember that we are not talking of a new strategy, given that since 2300 years ago, Susruta was the first one to use simulation, making incisions in a melon or with the use of linen dolls to practice dressings. However, current simulation must be well standardized and clearly cover the learning objectives. Simulation should have three fundamental stages:

\author{
Correspondence: \\ *Jesús Tapia Jurado \\ Academia Mexicana de Cirugía \\ Av. Cuauhtémoc $330,3^{\circ}$ Bloque B \\ Unidad de Congresos del Centro Médico Nacional \\ Siglo XXI \\ Col. Doctores \\ C.P. 06720, Ciudad de México, México \\ E-mail: tapiajj@ amcg.org.mx
}

Date of reception: 16-10-2017

Date of acceptance: 13-12-2017

DOI: 10.24875/CIRUE.M18000001
Cir Cir. 2018;86:1-3

Contents available at PubMed www.cirugiaycirujanos.com 
- Cognitive stage: in this stage, how, what for and why to work in the simulator and its educational rules will be known.

- Integrative stage: the student performs the maneuvers as many times as necessary until he/she dominates them.

- Autonomous stage: manual movements are already performed with dexterity and automatically.

We can add a fourth stage, which is evaluation, where demonstrating that the practiced skill was correctly learnt is intended, for which we need fair and objective evaluation systems.

There are different types of simulation. There is non-biological simulation, which is performed on inanimate objects of different materials, for example, a synthetic arm in which incisions and sutures are repeatedly made. There are more complex simulators, with computerized systems that enable them to recreate different pathological processes and even talk. There are also virtual simulators, especially for minimally invasive surgical skills, where basic bimanual and eye-hand depth exercises are performed, in addition to surgical procedures such as cholecystectomy, appendectomy, hysterectomy, prostatectomy or arthroscopy of the knee and shoulder. And there are even simulators for robotic surgery. Biological simulators can be made out of inert or hybrid animal segments (biological and non-biological), of pig-type live animal or corpse-like inert human material; the latter, thanks to the new preservation techniques, turns out to be a high-fidelity simulator, its use for training offers reality on human anatomy and tissue manipulation and, in addition, the same human inert model can be used for different procedures, although it has some disadvantages, such as the cost, absence of bleeding and rather limited availability ${ }^{6}$.

Numerous works have appeared over the past 10 years supporting the qualities of the use of simulation. For example, Draycott et al. ${ }^{7}$ compared, in a retrospective, observational, comparative study, shoulder dystocia problems secondary to a cesarean section, before and after training with simulation, showing that, prior to the course, $17 \%$ of newborns suffered brachial plexus excessive traction, and $7 \%$, of a lesion thereof, while in the group that received simulation, only $9 \%$ experienced excessive traction and the brachial plexus lesion decreased to $2.3 \%$. In laparoscopic surgery, residents who received simulation training through virtual models have been shown to acquire better quality in their surgical skills and abilities in comparison with those who did not practice simulation of any kind. It has also been demonstrated that, comparing residents who received simulation with endotrainer versus those who received simulation with virtual reality, the latter had significant improvements in their abilities ${ }^{8}$. In a work carried out by Cox, ${ }^{9}$ residents of three groups were compared: without simulation exercises, with virtual simulation and with simulation in an animal model. Those who did practice in an animal model achieved a shorter time to perform a laparoscopic cholecystectomy, and their surgical performance was superior to that of the other two groups, with decision making also being improved, demonstrating that the pig model provides fidelity and there is availability thereof; its disadvantages were high cost and anatomical differences. In neurosurgery ${ }^{10}$, using $40-\mathrm{kg}$ pigs, $97 \%$ of residents learned to handle complications, improved dissection of brain tissue and learned hemostasis and bleeding control by suction, concluding that the live-swine model offers an adequate experience for working on a pulsating and bleeding brain. In orthopedics ${ }^{11}$, simulations in synthetic models were shown to improve ability for arthroscopy, and it was concluded that simulation improves surgical performance in the real patient. Currently, the use of models with 3D printer has shown different advantages: the simulator is similar to the patient, the surgical act is practiced as many times as necessary, it enables the surgeon to plan the surgery and know possible complications and how to if the occur, and even how to avoid them; but as disadvantages there is the absence of bleeding, limited availability of textures with current materials and its elevated cost ${ }^{12}$.

With regard to finding a more accurate evaluation system by means of which we can confirm the acquisition of the taught skill, there are options, such as checklists and expert evaluation. With a two-camera system and marking the instruments that provide a computer record of movements, associated with mathematical analyses, movements were compared between medical undergraduate students, general surgery residents and expert surgeons with years of experience in laparoscopic surgery, which allowed objective evaluation of the following parameters: bimanual dexterity, trajectory length, perception of depth, acceleration, area energy and others. Thus, it was possible to see the number and quality of movements of each one of the evaluated subjects, whereby a more objective parameter is obtained to evaluate the acquisition of surgical skills in laparoscopy ${ }^{13}$. 
Needless to say, simulation for the acquisition of skills and abilities requires a complex and expensive infrastructure, which includes different surgical rooms, an animal facility with certified and trained staff to manipulate the living biological models and an amphitheater with current procedures for tissue preservation, which should comply with the regulations certificating and health institutions request; therefore, large simulation centers should be centralized in postgraduate educational institutions. It is important mentioning that, in these centers, not only surgical skills are acquired but, at the same time, the most current surgical procedures and the most innovative instruments and equipment are being known.

Finally, we will say that any simulation model is useful, regardless of how simple, sophisticated, cheap or expensive it is, and as long as we keep in mind what the learning purposes are. In addition, it has been demonstrated that surgical skills and abilities acquisition is faster, that education becomes the sole objective of the exercise and that simulation is an ethical necessity, since patients are protected and are not an educational object.

\section{References}

1. Makary M, Michael D. Medical error - the third leading cause of death in the US. BMJ. 2016;353:i2139.

2. Mattar SG. General surgery residency inadequately prepares trainees for fellowship: results of a survey of fellowship program directors. Ann Surg. 2013;258:440-9.

3. Tapia JJ. Los retos de la educación quirúrgica en el siglo XXI. Cir Gen. 2012;34:1-2.

4. Akhtar K, Chen A, Gupte S. The role of simulation in developing surgical skills. Curr Rev Musculoskelet Med. 2014;7:155-60.

5. Lazarini E, Schonstedt P, Abedrapo M, Yarmuch J, Csendes A, Rodríguez N. Simulación: una herramienta útil en la formación quirúrgica moderna. Rev Chilena de Cirugía. 2008;60:167-9.

6. Blackburn S, Griffin S. Role of simulation in training the next generation of endoscopist. World J Gastrointest Endosc. 2014;6:234-9.

7. Draycott T, Crofts J, Ash JP, Wilson LV, Yard E, Sibanda T, et al. Improving neonatal outcome tKhrough practical shoulder dystocia training. Obstet Gynecol. 2008;112:14-20.

8. Davis J, Khatib M, Bello F. Open surgical simulation- a review. J Surg Educ. 2013;70:618-27.

9. Cox M. Teaching surgical skills - changes in the wind. N Engl J Med. 2006;355:2664-9.

10. Regelsberger J, Eicker S, Siasios I, Hänggi D, Kirsch M, Horn P, et al. In vivo porcine training for cranial neurosurgery. Neurosurg Rev. 2015;38:157-63.

11. Akhtar K, Chen A, Gupte S. The role of simulation in developing surgical skills. Curr Rev Musculoskelet Med. 2014;7:155-60.

12. Waran VJ. Injecting realism in surgical training - initial simulation experience with custom 3D models. Surg Educ. 2014;71:193-7.

13. Pérez EF, Ordorica FRM, Oropesa Gl, Zalles VC. Face, content, and construct validity of the EndoVis training system for objetive assessment of psychomotor skills of laparoscopic surgeons. Surg Endosc. 2015;29:3392-403. 